\title{
IT-Projekte im deutschen E-Government: IT-Projektmanagement zwischen Planung und Selbstorganisation
}

\author{
Margit Scholl, Maika Büschenfeldt
}

Zusammenfassung

Die immer wiederkehrenden Probleme der Projektwirklichkeit in E-Government-Projekten belegen, dass dem Projektmanagement durch Vorabplanung und instruktive Intervention Grenzen gesetzt sind. Stark formalisierte und planungsdeterministisch ausgerichtete Entwicklungsmodelle sind bei starker Dynamik des sozialen Einsatzkontextes nicht die geeignete Lösungsstrategie zur Vermeidung des Scheiterns von (Software-)Projekten, sondern selbst Teil des Problems. Ein modernes, ganzheitliches, professionelles Projektmanagement sollte deshalb versuchen abzuwägen, wo eine gezielte Steuerung des Projektverlaufs und der Planungsvorgaben durch das Management noch gelten oder wo Entscheidungsbefugnisse an das Projektteam abgegeben werden sollten, um die nötigen Freiheiten für selbstorganisiertes Arbeiten zu schaffen, ohne dabei den Anspruch der Lenkung von Entwicklungsprozessen gänzlich aufzugeben.

\section{Abstract}

The recurring problems encountered in realising egovernment projects show that pre-planning and instructive intervention serve to set limits on project management. Where there are strong dynamics at work relative to the context of a (software) project's social application, highly formalized planning and deterministic models of development are not, in strategic terms, the most appropriate means of preventing its failure; in fact, they may even be part of the problem. Modern, holistic professional project management should therefore seek to assess whether management should control the project's progress and planning requirements or transfer decision-making powers to the project team in order to enable independent work, without giving up altogether the right to direct the processes of development.

\section{Einleitung}

Die Modernisierung von Staat und Verwaltung wird inzwischen maßgeblich durch den Einsatz und die Gestaltung moderner Informationstechnologien (IT) angetrieben und geprägt (Lucke 2005: 5) - mehr noch, IT wird zum »Motor für Veränderungen« und zur Triebfeder sozialer Innovationen in Staat und Verwaltung (Lucke 2005: 17). Anspruch des Projektmanagement ist es, Projekte so zu organisieren und zu steuern, dass die vorgegebenen Ziele innerhalb des gesetzten Zeit- und Kostenrahmens erreicht werden können. Die Einmaligkeit und Komplexität großer Entwicklungsprojekte stellen hohe Anforderungen an das Projektmanagement, weil nicht nur Innovatives geleistet werden muss, sondern die Komplexität des Vorhabens mit den vielen Teilprozessen viele Unsicherheiten und Projektrisiken birgt. So ist der Projektverlauf trotz dezidierter Projektplanung und sorgsam ausgearbeiteter Vorgehensmodelle in der Regel nicht eindeutig vorhersehbar. Hinzu kommt, dass aufgrund der komplexen Rahmenbedingungen die Anforderungen bei Projektbeginn zumeist unklar oder im Projektverlauf instabil sind (Miebach 2007). Da der erfolgreiche Einsatz von IT als erfolgskritisch für die Modernisierung von Staat und Verwaltung gilt, steht das moderne IT-Projektmanagement unter großem Erfolgsdruck, während die Umsetzung von IT-Projekten ausgesprochen risikobehaftet ist. In Abgrenzung zum Versuch, die Unsicherheiten in IT-Projekten durch aufwändige Planungsprozesse und instruktive Eingriffe aufzufangen, versucht dieser Beitrag Elemente der Selbststeuerung als adäquate Strategie der Problembewältigung unter instabilen und komplexen Umweltbedingungen einzuführen und theoretisch zu untermauern. Grundlage bildet der systemtheoretische Ansatz der Kontextsteuerung von Helmut Willke, der im Fol- 
genden auf die Grundprobleme des Managements komplexer IT-Projekte übertragen wird (Willke 1989; Willke 1996; Willke 1998). Grundidee dieses Ansatzes ist es, vorausplanende Elemente im Sinne einer vorgedachten Rationalität durch Auftraggeber, Vorgesetzte oder Verfahren (Fremdsteuerung) mit bewusst eingeräumten Gestaltungsfreiräumen (Selbststeuerung) der involvierten Mitarbeiter zu verbinden.

In Kapitel 2 werden die besonderen Rahmenbedingungen der öffentlichen Verwaltung als Umwelt von IT-Projekten eingeführt und die Bedeutung von IT für die Modernisierung von Staat und Verwaltung herausgestellt. Kapitel 3 führt in die wiederkehrenden Grundprobleme von IT-Projekten (der öffentlichen Verwaltung) ein. Auf dieser Grundlage wird in Kapitel 4 die Kontextsteuerung als alternativer Ansatz der Steuerung in komplexen und instabilen Umwelten vorgestellt. Im Sinne eines Ausblicks befasst sich Kapitel 5 mit dem Ausloten der Möglichkeiten zwischen institutionellem Rahmen des Managements und kreativer Selbstorganisation der Projektteams in der öffentlichen Verwaltung und der Frage, ob eine solche auch anerkannt und gefördert werden kann.

\section{Staat und Verwaltung als Umwelt von IT-Projekten}

Hermann Hill definiert E-Government als »Durchführung von Prozessen der öffentlichen Willensbildung, der Entscheidung und der Leistungserstellung in Politik, Staat und Verwaltung unter sehr intensiver Nutzung der Informationstechnik«. Diese Definition betont das erhebliche Potenzial des E-Governments hinsichtlich Transparenz, Kooperation und Interaktion aller involvierten Akteure und macht damit gleichzeitig deutlich, dass der öffentliche Sektor in einer Phase des radikalen Umbaus steht. E-Government-Projekte sind deshalb keineswegs »nur « komplexe IT-Projekte, sondern gehen in ihrem Anspruch und ihrer Wirkung weit darüber hinaus, da sie in letzter Konsequenz zu einen Umbau der Gesellschaft beitragen. Dies wird auch in der Projektorganisation im Verhältnis von (Linien-) Management und (Projekt-)Mitarbeitern, nicht ohne Folgen bleiben. [1]

Für die Bundesregierung und die öffentliche Verwaltung des Bundes als Großauftraggeber von Modernisierungsprojekten hat der Einsatz von IT eine neue Stufe erreicht: Ohne IT gibt es keine Modernisierung des
Verwaltungshandelns, keine Verbesserung der Dienstleistung und Servicequalität sowie keine Steigerung der Effizienz und Effektivität. Die Entscheidung für IT führt jedoch keinesfalls automatisch zum Erfolg, da der erfolgreichen Umsetzung von IT-Projekten häufig die traditionellen Strukturen der Verwaltung entgegen stehen. Die Beauftragte der Bundesregierung für Informationstechnik schreibt dazu: »Es ist nicht verwunderlich, dass die durch sehr kurze Innovationszyklen geprägte (IT-)Branche in der eher durch Beständigkeit geprägten Verwaltung auf eine gewisse Zurückhaltung stieß « (Roygall-Grothe 2010). Als erschwerend erweist sich dabei, dass traditionelles Verwaltungshandeln weniger an Leistungen und Ergebnissen orientiert sei, »sondern an Regeln und Verfahren in der Tradition ihrer hierarchischen Organisationsstrukturen und der funktionalen Arbeitsteilung« (Benedix 2009). Ausgehend von der Kritik an den zu starren Strukturen der Verwaltung wurde bereits durch den Leitbildwechsel der Prozessorientierung eine größere »Kundenbindung « in der öffentlichen Verwaltung erreicht. Notwendig wurde diese Form der Reorganisation durch Aufgaben, die mehr und mehr quer zu den traditionellen Verwaltungsabläufen stehen und veränderte Formen der Zusammenarbeit forderten. Dabei hat sich auch das Selbstverständnis der Verwaltung immer mehr von der Ordnungsbehörde in Richtung eines Dienstleisters entwickelt. Kommunen bezeichnen sich inzwischen als Bürgerkommunen und richten Dienstleistungscenter ein (Benedix 2009: 19). Der IT kommt dabei eine wichtige Rolle »als Instrument der Reorganisation « zu, die eine »Verbesserung interner Verwaltungsabläufe« ermöglicht und neue Kommunikationswege zwischen Verwaltung und Kunden erschließt (Seidel 2009). Insgesamt wird in dieser IT getriebenen Entwicklung eine deutliche Tendenz der Auflösung determinierender Abläufe zugunsten flexiblerer Strukturen sichtbar. Dennoch findet dieser Trend in den bereits vorhandenen Strukturen seine Grenzen. Das gilt insbesondere für die Bindung von Verwaltungshandeln an Recht und Gesetz, die Neuorientierungen in den legalistischen Anforderungen des Verwaltungshandelns begrenzt (Benedix 2009).

Bund, Länder und Kommunen arbeiten bereits daran, die strukturellen, technischen und rechtlichen Voraussetzungen für ein einheitliches E-Government zu schaffen. Der seit April 2010 etablierte IT-Planungsrat [2] hat im September 2010 dazu eine neue nationale E-Government-Stategie beschlossen [3]. Das war eine mehr als notwendige Maßnahme, um die deutsche E-Govern- 
ment-Stagnation im EU-Vergleich zu überwinden.[4] Im Vorfeld wurde dazu das Grundgesetz geändert. Es verankert nun mit Artikel 91c GG die Informationstechnik als eine der bedeutsamsten Infrastrukturen des 21. Jahrhunderts und schafft die rechtlichen Voraussetzungen für eine lückenlose und medienbruchfreie elektronische Kommunikation zwischen allen deutschen Behörden. Das für 2009 bis 2011 geltende Investitionsprogramm des Bundes von 500 Millionen Euro ist fast vollständig ausgegeben bzw. festgelegt und kam offenbar über 400 Unternehmen zugute.[5]

Aktueller Stand und notwendige Strategien insbesondere für ein wirtschaftsorientiertes E-Government wurden auf der Fachkonferenz »Wirtschaft und Verwaltung im Netz« diskutiert, die am 4. und 5. Oktober 2011 in Berlin stattfand.[6] Der aktuelle Stand des E-Governments in Deutschland verweist darauf, dass 40 Prozent der Deutschen, die im Internet aktiv seien, auch E-Government-Angebote nutzten, und nicht nur ein Mehr von E-Government-Angeboten gefragt ist, sondern Akzeptanz, Transparenz und Qualität von bestehenden Verwaltungsservices für Bürger und Unternehmen weiter zu erhöhen seien (Roygall-Grothe 2011). E-Government muss nach Cornelia Roygall-Grothe »in die Fläche gebracht werden«. Es seien dazu in etwa 3.500 Schriftformerfordernisse allein im Bundesrecht zu ändern, die der elektronischen Kommunikation im E-Government im Wege ständen und durch andere Verfahren zwar nicht ersetzt, aber ergänzt werden müssten. Im Forum A betonte dazu Martin Brüggemeier die Wichtigkeit, »dass Gesetze von Anfang an in Kenntnis und unter Beachtung des Verwaltungsvollzugs gemacht würden« und forderte desweiteren ein »Easy Government « (Brüggemeier 2011).

\section{Probleme des IT-Projektmanagements}

Das Bundesministerium des Innern (BMI) beschreibt die Projektwirklichkeit öffentlicher IT-Vorhaben als Problem rasch ansteigender Komplexität und konkurrierender Beziehungen der Projektzielgrößen wie Leistung, Zeit und Ressourcen.[7] Die Steigerung der Komplexität und des damit verbundenen Aufwands wird auf höhere Qualitätsanforderungen, zunehmenden Funktionsumfang, eine größere Anzahl von Beteiligten sowie die Integration von Alt-Verfahren und andere IT-Verfahren zurückgeführt (Ditzen 2009). Dieser Trend dürfte sich in den letzten zehn Jahren noch verstärkt haben, so- dass die Umsetzung von IT-Projekten zunehmend als kritisch und risikobehaftet einzuschätzen ist. Viele Autoren sehen deshalb ein professionelles Projektmanagement (PM) mit einer standardisierten und einheitlichen Vorgehensweise als den Schlüsselfaktor für die erfolgreiche Umsetzung von IT-Projekten an. Vor allem bei Großprojekten mit hohem Finanzvolumen, langen Laufzeiten und einer großen Anzahl von Beteiligten wird ein Mangel an professionellem Management als Ursache für katastrophale Fehlsteuerungen gesehen, die bis zum Projektabbruch führen können.

Vollständige Anforderungen sind ein elementarer Sachgegenstand für den Projektbeginn und eine zielorientierte Planung, die jedoch selten erfüllt sind und immer wieder zu Missverständnissen zwischen Auftraggebern und Auftragnehmern führten. So zeigen die bei Lamberts (2009) dargestellten Ergebnisse einer Studie aus dem Jahre 2004 unklare Ziele und unklare Anforderungen als Hauptursache für das Scheitern von Projekten. Es folgen fehlende Management-Unterstützung, eine mangelnde Projektmanagement-Methodik, Ressourcenmangel und mangelnde Qualifikation bzw. mangelndes Engagement. Die Ermittlung exakter Anforderungen fordert den beständigen Dialog zwischen Auftraggebern und Auftragnehmern und ein möglichst vollständiges Wissen um die Ziele, Rahmenbedingungen und Einflussgrößen des Projektes. Nach Erfahrungen der Autorinnen sehen sich einerseits viele Auftraggeber zumindest anfangs nicht in der Lage, ihre spezifischen Anforderungen klar und präzise zu formulieren, oder gehen sogar fälschlicherweise davon aus, dass es Aufgabe des Auftragnehmers sei, diese zu definieren. Andererseits gilt die frühzeitige Klärung der Anforderungen an Technik, Sicherheit und Qualität als Voraussetzung für die vertragliche Ausgestaltung, bestimmen Lieferumfang, Projekttätigkeiten und den Projekterfolg. Grundproblem bleibt, dass die Abschätzung des Aufwands und der Kosten häufig zu einem Zeitpunkt erfolgen muss, an dem man die Anforderungen und die Komplexität des Projektes noch gar nicht vollständig erfassen kann.

Ein weiterer Problembereich liegt in der unzureichenden organisatorischen Einbettung und mangelnden Akzeptanz in der Umsetzung von IT-Projekten der öffentlichen Verwaltung. So bemängelt der Bundesrechnungshof den unzureichenden Know-howTransfer in der öffentlichen Verwaltung und beklagt hinsichtlich der extern Beteiligten u. a. das fehlende Steuerungs-Know-how der öffentlichen Hand. Der Bun- 
desrechnungshof bestätigt in seiner Rückschau auch die fehlende Strategie der öffentlichen Verwaltung im Umgang mit Freistellungen, dem Vorhalten von Kompetenzen und der Qualifizierung des Projektpersonals. Nach wie vor fehlt eine angemessene Berücksichtigung von Technikkompetenz in der Ausbildung der Verwaltungsmitarbeiter (Ditzen 2009).

Die bislang noch ausstehende Ausprägung und Stärkung von Projektkompetenz bestätigt sich in den Ergebnissen einer Langzeitstudie von Gröger (2004), bei der Führungskräfte aus der deutschen Industrie, aus Dienstleistungsunternehmen und der öffentlichen Verwaltung befragt wurden. Der Studie zufolge wurden nur $13 \%$ der Projektarbeit als Wertsteigerung, 87\% hingegen als Wertvernichtung angesehen. Die Wertvernichtung durch Projektarbeit führt Gröger (2004) auf eine unzureichende Projektkompetenz zurück und fordert diese »stärker in den Fokus der Bemühungen des Topmanagements zu rücken«. Doch selbst wenn das Projektmanagement einen hohen Stellenwert besitzt, bedeutet dies keineswegs, dass das Topmanagement einen hohen Anteil seiner Arbeitszeit in projektverbundene Aktivitäten investiert, wie die Ergebnisse der Studie der European Business School (EBS) in Kooperation mit der Deutsche Gesellschaft für Projektmanagement (GPM) aus dem Jahr 2008 zeigen (EBS 2008). In dieser Studie gelten die Kompetenz und das Engagement der Projektmitarbeiter als größte Stärke. Eine weitere Studie der GPM in Zusammenarbeit mit der PA Consulting Group bestätigt dies und hält ebenfalls fest: »Kommunikation, Zieldefinition und die Qualifikation der Mitarbeiter bestimmen am häufigsten den Erfolg (oder Misserfolg) der ausgewählten Projekte.«(Engel 2008)

\section{Fremd- und Selbststeuerung im IT-Projektmanagement}

IT-Projekte lassen sich als einmalige und zielorientierte Vorhaben mit definiertem Anfang und Ende kennzeichnen. Ziele markieren die erwünschten Ergebnisse der Projektarbeit. Ihre wichtigste Funktion liegt in der Orientierung und Handlungskoordination. Da der Projektverlauf in der Regel nicht eindeutig vorhersehbar und damit auch nicht vollständig im Vorwege planbar ist, steht die Projektsteuerung für den Versuch, den Ablauf der Projektaktivitäten in einer gewünschten Weise zu beeinflussen, sodass die gesetzten Ziele innerhalb des vorgesehenen Kostenrahmens erreicht werden können (Breisig 2010; Willke 1998). Das Verhältnis von Steuerung und Planung und die Einschätzung der unterschiedlichen Managementstrategien, erschließt sich aus den leitenden Koordinationsmodellen. Helmut Willke unterscheidet idealtypisch zwei Leitmodelle der Handlungskoordination, die nicht nur für spezifische Aufbau- und Ablaufstrukturen der Organisation, sondern auch für unterschiedliche Typen der Steuerung stehen: Hierarchie »als allgemeines Modell der ungleichrangigen, fremdbestimmten und zentralisierten Koordination« und Demokratie als Modell »der gleichrangigen, selbstorganisierten und dezentralen Koordination" (Willke 1998: 89 f.). Als Strategie des Projektmanagements steht Hierarchie für eine feste Struktur der Arbeitsteilung, in der Planung, Ausführung und Zuständigkeiten klar voneinander getrennt sind. Kontrolle, Planungs- und Weisungsbefugnis verlaufen entsprechend der hierarchischen Rangfolge von oben nach unten. Aus der Perspektive der ausführenden Projektmitarbeiter dominiert damit die Fremdsteuerung durch Auftraggeber, Vorgesetzte oder Verfahren. Für die Projektmitarbeiter ist dies mit eher geringen Entscheidungsspielräumen in der Projektarbeit verbunden. In einem demokratischen Projektmanagement hingegen dominiert Selbststeuerung im Sinne einer dezentralen und gleichrangigen Verteilung der Entscheidungsfähigkeit und -befugnis. Da die festen Grenzziehungen zwischen Über- und Unterordnung, abhängiger und unabhängiger Arbeit sowie Planung und Ausführung fehlen, verbleibt die Handlungsautonomie weitestgehend bei den ausführenden Projektarbeitern.

In der Gegenüberstellung beider Koordinationsmodelle fällt auf, dass die Stärken und Schwächen beider Modelle miteinander korrespondieren. Es wird deutlich, dass Stabilität und Orientierung in einem Spannungsverhältnis zur Flexibilität und Innovationskraft stehen und dass beide Modelle dieses Spannungsverhältnis in ihren Stärken und Schwächen verkörpern.

So bietet ein hierarchisches Projektmanagement zwar Stabilität und Orientierung, gilt aber wohl gerade deshalb als unflexibel, bevormundend und innovationsfeindlich, während sich die Demokratie als kreatives und flexibles Modell bewährt, aber auch der Befürchtung Nahrung bietet, dass die Freiheiten radikal-demokratischer Kooperation im Chaos münden und Projekte dem Diktat unqualifizierter Laien unterworfen werden. Das demokratische Modell weist zwar einen geringen Steuerungs- und Planungsaufwand auf, stellt aber hohe Anforderungen an die Qualifikationen 


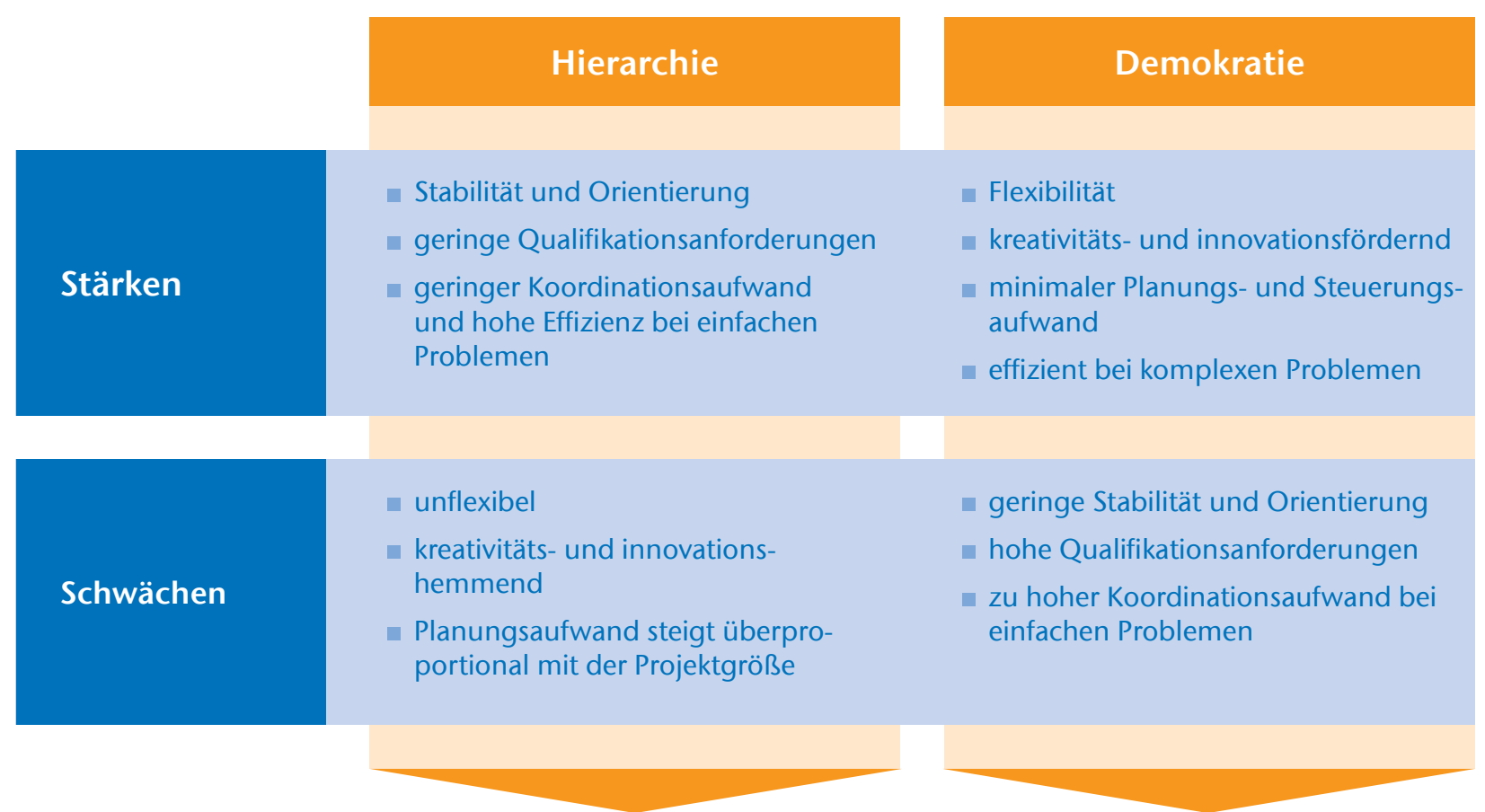

Risiko!

Überregelung | Übersteuerung Unterregelung | Überforderung

Abb. 1: Stärken und Schwächen des hierarchischen und demokratischen Koordinationsmodells

und Erfahrungen der beteiligten Akteure. Fehlen die zur Selbststeuerung notwendigen Kompetenzen und Qualifikationen auf der ausführenden Ebene (Projektmitarbeiter), wird die Zurückhaltung in den Vorgaben leicht zur Überforderung. Im hierarchischen Modell ist dieses Problem weniger virulent, weil formale Regeln, rigide Planung und instruktive Intervention jene Stabilität und Orientierung bieten, die es gestatten, auch gering qualifiziertere oder unerfahrene Projektmitarbeiter zu beschäftigen. Durch die Trennung von Planung und Ausführung sind in diesem Modell Erfahrungen, personale Kompetenzen und Qualifikationen nur im Management notwendig, nicht aber auf der ausführenden Ebene - ob damit allerdings die geforderte Projektund Produktqualität tatsächlich erreicht werden kann, sei dahingestellt. Es ist in diesem Modell auch viel leichter, bewährtes Prozess- und Methodenwissen zu konservieren und über Vorgehensmodelle, Richtlinien, Methodenbeschreibungen, Handlungsanweisungen etc. weiterzugeben. Mangelnde Projekterfahrung und geringe Qualifikation sind bei einem guten Methodenrepertoire, guter Planung und zielführender Intervention durch das Management grundsätzlich unproblematisch. Dafür ist ein hierarchisches Projektmanagement mit dem Projektrisiko der Übersteuerung bzw. Überregelung verbunden. Symptomatisch dafür ist die aus der beobachtbaren Projektwirklichkeit ableitbare Erkennt- nis, dass die Wahrscheinlichkeit des Scheiterns in engem Zusammenhang mit der Komplexität der Projekte steht. Dabei gilt, je aufwändiger die Projektplanung ist, desto größer ist das Risiko des Scheiterns (Dietrichs 2004: 5f.; Chaos Report 2004). Hier kommt zum Tragen, dass der Aufwand für die Planung und das Management des Projektverlaufs überproportional zur Projektgröße ansteigt und damit selbst einen Beitrag zur Unüberschaubarkeit und Komplexität der Projekte leistet.

Mit Bezug auf die Koordinationsmodelle hängt die Einschätzung einer angemessenen Strategie im konkreten Umgang mit den wiederkehrenden Problemen in IT-Projekten davon ab, wo die Ursachen für das Scheitern vieler Projekte zu suchen sind, in einem zu starken oder zu geringen Reglement der Fremdsteuerung. Elemente der Fremdsteuerung sind auch Vorgehensmodelle, die mit dem Anspruch entwickelt werden, für die immer wiederkehrenden Probleme der Softwareentwicklung wiederholbare Lösungskonzepte anzubieten. So stellen Moll u. a. (2004) fest, dass 50 Prozent aller Unternehmen ISO-zertifizierte Vorgehensmodelle oder Quasi-Standards einsetzen und dennoch 74 Prozent aller Software-Projekte nicht uneingeschränkt erfolgreich abgewickelt werden können. Sie folgern daraus, dass »die Umsetzung dieser Standards in der industriellen Praxis entweder nicht den gewünschten Erfolg bringen oder nicht wirklich stattfinden« (Moll 2004: 425f.). 
Softwareprojekte können somit scheitern, weil die bereits entwickelten Prozesse, Werkzeuge, Methoden und Standards nicht angewendet werden oder im Gegenteil das entwickelte Instrumentarium nicht geeignet ist, um den gewünschten Erfolg zu bringen.

Systemtheoretische Denkansätze verweisen mit dem Hinweis auf die Kontra-Intuitivität (Willke 1996; Willke 1998) des sozialen Kontextes auf die letzte Option mit dem Hinweis, dass die Ursachen und Wirkungen in komplexen Projekten nicht linear-kausal, »sondern räumlich und zeitlich, sachlich und kognitiv variabel und verwickelt verbunden « sind (Willke 1998). Das Problem unklarer Anforderungsdefinitionen gehört damit zum Wesen von IT-Projekten, da die starke Abhängigkeit zwischen IT-Einsatz und sozialem Kontext eine präzise, eindeutige, widerspruchsfreie und stabile Anforderungsdefinition gar nicht zulässt. Softwareentwicklung schließt in dieser Perspektive die Auseinandersetzung mit der eigenwilligen Dynamik sozialer Systeme ein und trägt dem Umstand Rechnung, dass exakte Anforderungsspezifikationen und detaillierte Planungsvorgaben häufig nur eingeschränkt oder unter Umständen gar nicht möglich sind. Stark formalisierte und planungsdeterministisch ausgerichtete Entwicklungsmodelle wären bei starker Dynamik des sozialen Einsatzkontextes dann nicht die geeignete Lösungsstrategie zur Vermeidung des Scheiterns von Softwareprojekten, sondern Teil des Problems. Deutlich wird dies, wenn man bedenkt, dass Methoden der Komplexitätsbewältigung und Kontrolle in stark formalen (und damit wiederholbaren) Entwicklungsmodellen, wie Spezialisierung, Planung, Controlling, Entwurf, Dokumentation und Vertragswerken auch den Aufwand steigern und damit selbst Komplexität erzeugen. Ein modernes, ganzheitliches, professionelles Projektmanagement setzt deshalb dort an, wo Vorgehensregeln und Planungsdeterminismus nicht greifen oder gar versagen und ein Vorgehensmodell Lücken lassen muss.

Das eigentliche Risiko einer plandeterminierten Steuerung besteht darin, dass vorgedachte Lösungen nicht zu den Problemen und Anforderungen eines dynamischen und komplexem Umfeldes passen. Hier zeigt sich, dass die plandeterminierte und zentralisierte Steuerung im Projektalltag häufig nicht mehr zu leisten vermag, was einst als ihre Stärke galt, »nämlich Entlastung und Effizienzsicherung zu gewährleisten« (Breisig 2010: 23). Die Alternative liegt in Konzepten der Selbststeuerung, die darauf setzen, dass Projektmitarbeiter anfallende Probleme und Anforderungen flexibel und autonom in
Eigenregie zu lösen vermögen. Dennoch ist Steuerung durch Zielvorgaben und Planung nicht nur restriktiv, sondern macht Dinge häufig erst möglich. Nach wie vor ist die effektive und effiziente Zusammenarbeit vieler Menschen in großen Projekten mit komplexen Aufgabenstellungen nur durch Elemente der Planung und formale Strukturen denkbar. Hinzu kommt, dass der institutionelle Rahmen die Berücksichtigung formaler Vorgaben erfordert. Die eigentliche Frage ist damit, wo die sinnvolle Grenze zwischen Fremd- und Selbststeuerung verlaufen sollte.

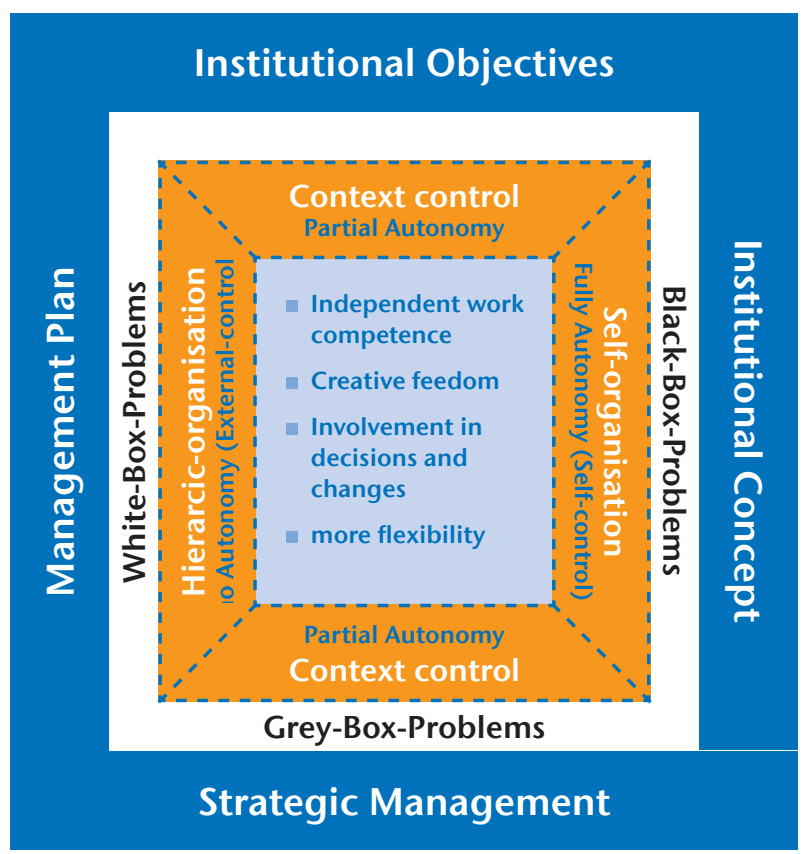

Abb. 2: Mögliche Steuerung in der Organisation: Plandeterminierte Vorgaben bilden den äußeren Rahmen (blauer Bereich), über Kontextsteuerung (gestrichelter Rahmen) könnten Rahmenbedingungen problembezogen die Arbeitspotenziale der Projektteams steuern, bis hin zum einzelnen Projektmitarbeiter (das Individuum ist im Mittelpunkt der Abbildung zu denken). Die Abbildung entstand als eigenständige, zusammenfassende Interpretation des Buches von Breisig (2010) unter Berücksichtigung der Ausführungen von Willke (1996).

In der Systemtheorie wird der Balanceakt zwischen Fremd- und Selbststeuerung unter dem Begriff der Kontextsteuerung als Mischform beider Steuerungstypen, d. h. der Fremd- und Selbststeuerung konzeptualisiert. Grundidee ist, dass der Grad einer planenden Einflussnahme von der Beschaffenheit der zu behandelnden Probleme abhängig gemacht wird. Die Einschätzung der Angemessenheit von Formalismen und Planung wird damit abhängig vom Grad der Problemkomplexität. In Anlehnung an Hellmut Willkes Interventionstheorie lassen sich grob drei Problemtypen unterscheiden (Willke 1996: 211ff.): 
- White-box Probleme sind von einfacher Beschaffenheit, da die Umwelt klar strukturiert, transparent und stabil ist. Eine exakte Planung ist hier problemlos möglich, da Ursache und Wirkung in einem linearkausalen Zusammenhang stehen und die Reaktion auf Einflussnahmen vorhersehbar (intuitiv) ist.

- Grey-box Probleme präsentieren sich als Grauzone durchschaubarer und nicht durchschaubarer Strukturen. Planung ist hier eingeschränkt möglich, bleibt aber von Unsicherheiten begleitet.

- Black-box Probleme stellen den schwierigsten Fall dar, da die Umwelt komplex und instabil ist. Eingriffe und Vorgaben lösen kontra-intuitive, d. h. nicht vorhersehbare Reaktionen aus. Die Unsicherheit ist hier sehr groß, da unklar bleibt, wie sich Eingriffe oder Steuerungsversuche auswirken.

Im ersten Fall bietet die exakte Ausarbeitung einzelner Prozessschritte und stark formalisierter Entwicklungsmodelle große Vorteile, weil sie eine klare Struktur für die abgestimmte und arbeitsteilige Abarbeitung der einzelnen Projektphasen bietet und eine gute Planung ermöglicht. Ein Vorgehen dieser Art funktioniert jedoch nur, wenn sich analytische und synthetische Phasen klar voneinander abgrenzen lassen, d. h. wenn sich Anforderungen, Leistungen und Abläufe in der Planungsphase auch präzise beschreiben lassen. Die in Kapitel zwei und drei beschriebenen Rahmenbedingungen und Probleme in der Umsetzung von E-GovernmentProjekten in Deutschland lassen aber weder auf eine einfache Problembeschaffenheit, noch auf eine stabile Umwelt schließen. Die IT getriebene Modernisierung von Staat und Verwaltung kommt in Deutschland einer Revolution gleich, die einen tiefen Eingriff in die bisherigen Strukturen der Verwaltung und Politik darstellt und vielschichtige Interessen betrifft. Da diese Eingriffe das Handeln in Politik und Verwaltung nicht nur optimieren, sondern auch radikal verändern, ist mit der Umsetzung von E-Government-Projekten auch soziale Innovation im Sinne des Umbaus von Staat und Gesellschaft verbunden.

Die Entwicklung von E-Government-Projekten der Verwaltung wird deshalb nicht nur flexibel auf Unsicherheiten, wechselnde Anforderungen und unterschiedliche Interessenlagen zu reagieren haben, sondern auch Raum für Innovationen bieten müssen. Die Praxis des Projektmanagements in der öffentlichen Verwaltung wird deshalb mit dem zweiten und dritten Problemtyp zu kämpfen haben. Die Kontextsteuerung bietet hier die Möglichkeit, den Unsicherheiten durch eine Zurückhaltung in der Planung zu begegnen. Die Funktion des Managements und der Projektsteuerung wird sich auf das Setzen von Rahmenbedingungen beschränken, um damit den nötigen Raum für »die produktiven Selbststeuerungskräfte« zu schaffen (Breisig 2010: 23). Das darf nicht bedeuten, dass sich das (Top-) Management der öffentlichen Verwaltung seiner Verantwortung entzieht. Vielmehr bedeutet Kontextsteuerung das bewusste Setzen von Rahmenbedingungen für erfolgreiche Projektteams, das strategische Schaffen von Freiheiten für eine selbstorganisierte und dennoch zielorientierte Arbeit. Der Anspruch auf Lenkung von Entwicklungsprozessen und die Verantwortung seitens des Managements wird dabei nicht völlig aufgegeben. In der neueren Managementliteratur und der Unternehmensorganisation wird ein Umdenken in diese Richtung bereits erkennbar. Zwischen den Polen radikal dezentraler Koordination und des Planungsdeterminismus zeichnen sich seit Jahren folgende Tendenzen ab (siehe dazu auch Willke 1989):

- der Abbau hierarchischer Ebenen und die Verlagerung von Kompetenz und Verantwortung in autonome Arbeits- oder Projektgruppen,

- die wachsende Bedeutung flexibler Strukturen zulasten stabiler Strukturen,

- die wachsende Bedeutung integrativer Mechanismen und Rollen,

- der Wandel unternehmerischer Leitungsaufgaben zur Stützungsfunktion für selbstorganisierte Prozesse.

Hinsichtlich der öffentlichen Verwaltung in Deutschland bleibt die Notwendigkeit festzuhalten, dass die Entwicklung von E-Government mit der Entwicklung einer strategischen Ausrichtung des Projektmanagements mit einer Kontextsteuerung verbunden sein muss.

\section{Ausblick auf Ansätze einer Reform für E-Government-Projekte}

E-Government-Projekte dürften ein fester Bestandteil der öffentlichen Verwaltung bleiben und in Zukunft eine immer bedeutendere Rolle spielen. Ein Ansatz der Kontextsteuerung könnte helfen, den typischen Problemen der Umsetzung von IT-Projekten zu begegnen, stellt aber gleichzeitig einen radikalen Bruch mit dem traditionell bürokratischen Aufbau der Verwaltung dar. Problematisch bleibt, dass die Selbststeuerung hohe Anforderungen an die Mitarbeiter stellt und die umge- 
benden Organisationsstrukturen dieses auch zulassen müssen. In dieser Hinsicht ist die öffentliche Verwaltung noch schlecht gerüstet. Mit Bezug auf die Funktion des Managements stellen sich dabei die folgenden Fragen:

- Wird Wesentliches für innovative Projekte durch das Management der öffentlichen Verwaltung in seiner Innenstruktur tatsächlich erreicht?

- Gelingt es dem Management, die Voraussetzungen dafür zu schaffen, dass Projekteteams gegebene Ziele selbstständig erreichen können?

- Werden organisatorische und emotionale Hürden tatsächlich abgebaut?

- Stärkt die Führung die Projektmitarbeiter und motiviert es diese mit Vertrauen und Unterstützung?

In Beantwortung dieser Fragen wird deutlich, dass hier noch erheblicher Handlungsbedarf besteht. Festzuhalten ist zunächst, dass es in der öffentlichen Verwaltung und in Unternehmen eine deutliche Diskrepanz zwischen Theorie und Praxis gibt. Bolte u. a. (2008: 19) führen dazu aus, dass Projektarbeit »als außerordentlich selbständige, kreative, kooperative und kommunikative Arbeit mit hohen Autonomiespielräumen« gilt, die »tatsächliche Ausgestaltung, organisatorische Verankerung und Arbeitsweise einer Projektgruppe (...) jedoch von Unternehmen zu Unternehmen und auch von Projekt zu Projekt stark variieren«. Annegret Bolte weist ferner darauf hin, dass die Theorie zur optimalen Projektarbeit und die Praxis in den Unternehmen oftmals weit auseinanderliegen und die entscheidende Frage nach den tatsächlichen Handlungsspielräumen durch die Selbstorganisation in den Projektteams nach wie vor offen bleibt.

Dies spiegelt sich auch im Forschungsbedarf wider, den eine GPM-Studie (Spang 2009) auf der operativen Ebene hinsichtlich des Ressourcenmanagements, des Störungs- und Krisenmanagements sowie der Mitarbeitermotivation ausweist. Auf der Integrationsebene ist im Hinblick auf unseren Beitrag bemerkenswert, dass das Team- und Kommunikationsmanagement zwar Interesse genießt, eine optimale Anwendung in der Praxis jedoch noch nicht erfolgt ist. Auf der strategischen Ebene sehen die Experten Forschungsbedarf in der Beurteilung der strategischen Bedeutung von Projekten und der organisationalen Strategieentwicklung. Im Personalbereich wird der Forschungsbedarf weniger im Personalmanagement aber deutlich in der Personalentwicklung gesehen. Sogar bezogen auf Grundelemente der Projektarbeit wie dem Projektmanager werden Bedarfe formuliert, vor allem im Bereich Führungsstil und Führungskompetenz. Auch für die Arbeitswissenschaften als Element des Projektmanagements wird Forschungsbedarf angemeldet, zumal dieser in der Literatur - laut Studie - noch nicht so häufig thematisiert wurde.

Für wichtig halten wir deshalb, dass das Management die Aufgabe der institutionalisierten Rahmensetzung wahrnimmt und die Voraussetzung für die kreative Arbeit der Projektleitung und des Projektteams bewusst schafft. Dazu müssten innovative E-Government-Projekte auf allen Verwaltungsebenen - Bund, Länder, Kommunen - als Chefsache begriffen werden. Chefsache meint im Sinne der Kontextsteuerung, dass das Management der öffentlichen Verwaltung einen Rahmen für das kreative Arbeiten der Projektteams setzt, ohne sich der eigenen Verantwortung zu entziehen, und damit diesen Rahmen sichert. Verantwortung und Kompetenz werden in autonom agierende Teams verlagert, deren Rücken durch die Projektleitung und das Management freigehalten werden. Dies würde die notwendigen Handlungs- und Gestaltungsspielräume schaffen, um innovative Lösungen zu finden und den Unsicherheiten in komplexen IT-Projekten zu begegnen. Es macht IT-Projekte flexibel und schafft Akzeptanz, ohne den Anspruch der Lenkung durch das Management aufzugeben. In diesem Sinne hätte das Management der öffentlichen Verwaltung als öffentlicher Auftraggeber die Aufgabe, die Institutionalisierung eines professionellen Projektmanagements mit u. a. einer einheitlichen Dokumentation, einem gezielten Multiprojektmanagement (MPM) und einer schlüssigen Auswahl der Projekte und Zuordnung der Mitarbeiter sowie einer überlegten Kompetenzübertragung an die Projektleitung zu sichern. Ein modernes MPM müsste dabei nach Wischhusen (2010) auf einem System basieren, das zeitgemäße, integrierte Managementmethoden nutzt. Die Gefahr an unzureichend organisatorischer Einbettung und mangelnder Akzeptanz ist damit allerdings noch nicht aus dem Weg geräumt. Vielmehr sollten solche Aspekte durch eine vorausschauende und intensive Projektvorbereitung mit klarer, ganzheitlicher Zieldefinition unter reflektierender Berücksichtigung der sog. Stakeholder und Risikoanalysen mit Präventivmaßnahmen berücksichtigt werden. Dies erfordert allerdings Zeit, deshalb erscheint es entscheidend, dass sich der öffentliche Auftraggeber seiner eigenen Rolle bewusst wird und wissen sollte, welche Kompetenzen intern vorzuhalten sind, um seine Beteiligung und Kontrollfunktion annehmen zu können. 
Die Verlagerung von Autonomie und Gestaltungsspielräumen in die Projektteams erfordert deutliche Veränderungen in den verwaltungstypischen Aufgabenprofilen und Berufsbildern. Ziel dieser Veränderungen wird sein, die Mitarbeiter so vorzubereiten und zu schulen, dass diese den Anforderungen an Selbstständigkeit, technischer Kompetenz, Flexibilität, Verantwortung, Koordinations- und Kommunikationsfähigkeit gerecht werden können. Zusätzlich muss dafür Sorge getragen werden, dass auch hinreichende Personalkapazitäten zur Verfügung stehen. Nach unseren Erfahrungswerten gelingt es gerade in Zeiten des ständigen Personalabbaus im deutschen öffentlichen Dienst immer weniger, die nötigen qualifizierten Ressourcen für temporäre Projekte zur Verfügung zu stellen. Projekte werden in öffentlichen Verwaltungen nicht selten »nebenbei« gemacht, erhalten so vom Management nicht die notwendige Bedeutung und führen bei Engagement der Projektleitung und der Mitarbeiter zu erheblichen Mehrbelastungen des Projektteams, ohne dass dies tatsächlich vom Management honoriert wird. Hinzu kommt die Frage, welche Kompetenz der Projektleitung zugesprochen wird? Wenn es sich nicht um politisch motivierte Projekte handelt, die eher zu einer reinen Projektorganisation und Weisungsbefugnis der Projektleitung führen, bleibt im Verwaltungsalltag organisatorisch i. d. R. alles wie es ist und die Verwaltungsmitarbeiter werden $z u$ sätzlich zu ihrer Verwaltungstätigkeit zu Projektleitern - und zwar ohne Weisungsbefugnis und tatsächlich zugesprochene Kompetenz und ohne eine entsprechend rechtzeitig durchgeführte Qualifizierung. Hier kann sicherlich ebenfalls ein Grund für das Scheitern von kleineren und mittelgroßen Projekten der öffentlichen Verwaltungen in Deutschland vermutet werden. Nur bei ganz großen Projekten wird die Weisungsbefugnis bisher beachtet.

Als Konsequenz müsste das (Top-)Management der öffentlichen Verwaltung in Deutschland seine internen Projektteams in vielfältiger Weise konsequent stärken, ihnen Zeit und Kompetenzen einräumen sowie die Entwicklung eines neuen Fähigkeitsprofils unterstützen, dessen Ausgestaltung mit dem Begriff »Kontextsteuerung«im engen Zusammenhang steht.

\section{Anmerkungen}

[1] Bemerkenswerte Erfahrung der E-Government-Projekte der ersten Stunde (Jahre 2000 - 2005) war, dass sie »Chef-Sache« sein müssen, d. h., dass das Top-Management der öffentlichen Verwaltung eine für den Erfolg der Projektarbeit begünstigende Umweltbedingung setzen muss. Siehe: E-Government Handbuch, OnlineVersion https://www.bsi.bund.de/DE/Themen/EGovernment/ EGovernmentHandbuch/OnlineVersion/onlineversion_node. html, Zugriff 30.10.11.

[2] Informationen zum IT-Planungsrat finden sich auf der Website der Beauftragten der Bundesregierung für Informationstechnik, http://www.cio.bund.de/cln_093/DE/Ueber_uns/IT-Planungsrat/ it-planungsrat_node.html, Zugriff 18.01.2011.

[3] Nationale E-Government-Strategie: Beschluss des IT-Planungsrats vom 24. September 2010, http://www.cio.bund.de/SharedDocs/ Publikationen/DE/Aktuelles/nationale_e_government_strategie_beschluss_20100924_download.pdf?_blob=publicationFile, Zugriff 08.01.2011.

[4] Vgl. hierzu die Abbildung nach http://lh4.ggpht. com/_3uCQVvx80II/TAqsiZ3EVYI/AAAAAAAACp4/bauAuxdsc0/egovernment-entwicklung-2010.jpg, Zugriff 16.01.2011.

[5] IT-Investitionsprogramm - Wichtiges Etappenziel erreicht: http://www.cio.bund.de/SharedDocs/Kurzmeldungen/ DE/2011/20110114_it_investitionsprogramm.html, Zugriff 08.01.2011.

[6] http://www.CIO.bund.de/SharedDocs/Kurzmeldungen/ DE/2011/20111026_fachkonferenz_witschaft_und_verwaltung. html, Zugriff 26.10.2011.

[7] Dies geschah 2001 auf Nachfrage des Bundesrechnungshofs (BRH) nach »Verbesserungspotentialen bei IT-Großprojekten« bzw. bei »Software-Entwicklungsprojekten«.

\section{Literatur}

Benedix, J. u. a. (2009): Herausforderungen mit neuen Organisationsformen meistern. In: Innovative Verwaltung, Ausgabe 11/12,1922.

Bolte, A. u. a. (2008): Die alltägliche Last der Kooperation - Abstimmung als Arbeit und das Ende der Meeting-Euphorie, edition sigma, Berlin.

Breisig, T. (2010): Unternehmenssteuerung - eine konzeptionelle Einführung. Ansätze - Methoden - Akteure, edition sigma, Berlin.

Brüggemeier, M. (2011): »Easy Government« - Bürokratieabbau im Verwaltungsvollzug durch IKT. Beitrag und Präsentationsfolien im »Fachforum A: Prozesse zwischen Wirtschaft und Verwaltung «, Fachkonferenz »Wirtschaft und Verwaltung im Netz«, 4. und 5. Oktober 2011, Berlin, http://www.dihk.de/branchen/ informations-und-kommunikationsbranche/e-government/egovern ment/wirtschaft-und-verwaltung-im-netz/fachforum-a, Zugriff: 29.10.2011.

Chaos Report (2004): Standish Group.

Dietrichs, H. (2004): Komplexitätsreduktion in der Softwareentwicklung - Ein systemtheoretischer Ansatz. Books on Demand, Norderstedt.

Ditzen, U. (2009): Verbesserungspotentiale bei der Umsetzung von (IT-) Projekten. Bericht über eine Qerschnittsuntersuchung des Bundesrechnungshofes 2005-2008, Präsentation im BAköV ITForum »Projektmanagement und IT-Bund «, 13.06.2009 in Berlin. 
EBS (2008): Potentiale und Bedeutung des Projektmanagements aus der Perspektive des Topmanagements. Studie der European Business School EBS in Kooperation mit der GPM, http://www. gpm-ipma.de/fileadmin/user_upload/Know-How/Studienergebnisse_Top-Manage ment.pdf, Zugriff: 17.02.2011.

Engel, T. u. a. (2008): Projektmanagement Studie 2008 - Erfolg und Scheitern im Projektmanagement,Gemeinsame Studie der GPM und der PA Consulting Group, http://www.gpm- ipma.de/ fileadmin/user_upload/Know-How/Ergebnisse_Erfolg_und_ScheiternStudie_2008.pdf, Zugriff 17.02.2011.

Gröger, M. (2004): Projektkompetenz: Mangelhaft. Stolperstein auf dem Weg zum Unternehmenserfolg, http://www.twinn.de/ leadership04/pdf/groeger_pbm.pdf, Zugriff: 17.02.2011.

Hill, H. (o. J.): eGovernment - Mode oder Chance zur nachhaltigen Modernisierung der Verwaltung?, Speyer, http://www.dhv-speyer. de/hill/Publikationen/E-Gov.pdf, Zugriff: 08.01.2011.

Lamberts, R. (2009): Projektmanagement in der öffentlichen Verwaltung. In: Becker, L.: Projektführung und Projektmanagement, Symposium Publishing GmbH.

Lucke von, J. u. a. (2005): E-Government-Forschungsplan: Handlungsfelder für eine neue Strategie in Deutschland. Gesellschaft für Informatik (GI), Fachbereich Rechts- und Verwaltungsinformatik Fachausschuss Verwaltungsinformatik (VI), Bonn.

Miebach, B. (2007): Organisationstheorie, Problemstellung - Modelle - Entwicklung. VS Verlag für Sozialwissenschaften, Wiesbaden.

Moll, K.-R. u. a. (2004): Erfolgreiches Management von SoftwareProjekten. In: Informatik-Spektrum.

Rogall-Grothe, C. (2010): Potenziale des E-Governments gemeinsam nutzen. In: eGovernment Kompendium 2011 - IT-Referenzbuch für den Öffentlichen Sektor, Vogel Business Media, Würzburg.

Rogall-Grothe, C. (2011): Keynote zum aktuellen Stand des EGovernments in Deutschland und Auszug auf Video, Fachkonferenz »Wirtschaft und Verwaltung im Netz«, 4. und 5. Oktober 2011, Berlin, http://www.dihk.de/branchen/informations-undkommunikationsbranche/e-go vern ment/e-government/wirtschaft-und-verwaltung-im-netz, Zugriff: 29.10.2011.

Seidel, A. u. a. (2009): Zukunftsreport Moderner Staat 2009 - Prozessmanagement im Jahr 2020. In: Special Innovative Verwaltung, Ausgabe 11/12, 2009.

Spang, K. u. a. (2009): GPM-Studie 2008/2009 zum Stand und Trend des Projektmanagements, Deutsche Gesellschaft für Projektmanagement e.V. (GPM), Universität Kassel, Fachgebiet Projektmanagement, http://www.gpm-ipma.de/fileadmin/user_upload/ Know-How/00-Gesamt-Studie-GPM-Juli_2009.pdf, Zugriff: 17.02.2011.

Willke, H. (1989): Controlling als Kontextsteuerung - Zum Problem dezentralen Entscheidens in vernetzten Organisationen, In: Eschenbach, Rolf (Hg.): Supercontrolling - vernetzt denken, zielgerichtet entscheiden. WUV, Wien.

Willke, H. (1996): Systemtheorie II. Interventionstheorie, Lucius \& Lucius, Stuttgart.

Willke, H. (1998): Systemtheorie III. Grundzüge einer Theorie der Steuerung komplexer Sozialsysteme, 2. ed., Lucius \& Lucius, Stuttgart.

Wischhusen, J. (2010): Anforderungen an ein modernes Multiprojektmanagement in der Bundesbehörde des Bundesamtes für Verbraucherschutz und Lebensmittelsicherheit (BVL). Master Thesis an der Paritätischen Akadamie gGMBH.

\section{Autoren}

Prof. Dr. Margit Scholl

Fachbereich Wirtschaft Verwaltung und Recht

Technische Hochschule Wildau [FH]

margit.scholl@th-wildau.de

\section{Dr. Maika Büschenfeldt}

Fachbereich Wirtschaft Verwaltung und Recht Technische Hochschule Wildau [FH]

maika.bueschenfeldt@th-wildau.de 